\title{
La Théologie du Rapprochement: Le Christianisme Catholique Contemporain et le Dialogue avec 1'Islam
}

\author{
Prof. Dr. Mahmoud HAIDAR ${ }^{(*)}$ \\ Président du Centre \\ Delta pour la Presse et \\ la Recherche \\ Approfondie - Beyrouth
}




\section{Introduction}

La relation entre les religions, et notamment le christianisme et l'islam, n'a pas emprunté un mode historique unique. De nombreux facteurs, composés, théologiques, doctrinaires, culturels et civilisationnels, l'ont placée dans des espaces mouvants, progressant parfois et reculant parfois, ou empruntant le chemin du dialogue et de l'interaction positive, d'autres fois.

Comment percevoir la relation entre le christianisme et l'islam, à partir de cette hypothèse, et peut-on élaborer une stratégie du dialogue et du rapprochement sur la base de la croyance en l'unité de la source divine pour les deux doctrines, chrétienne et musulmane ? Cette question constitue l'axe de notre étude qui tente de présenter la conception théologique contemporaine du christianisme catholique de ce qu'on appelle "la théologie du rapprochement». Tout comme elle tente d'exposer une vision globale de l'islam et des musulmans, à partir de la doctrine $\mathrm{du}$ salut, nous appuyant pour notre analyse sur les textes du second concile pontifical (1962-65) qui a subi des transformations au niveau de la connaissance, de la doctrine et de la théologie, qui ont eu un impact profond sur la vision du christianisme catholique vis-à-vis des autres religions et croyances mondiales, et notamment l'islam.

Dans ce cadre, nous avons abordé le mouvement d'interprétation que l'Eglise a connu à propos de la doctrine du salut, notamment en ce qui concerne la relation avec les autres religions, à savoir si leurs fidèles bénéficieraient de la grâce du salut représenté par la personne même du Christ. Notre étude porte donc, en premier lieu, sur le niveau atteint par la pensée théologique chrétienne envers l'islam et en second lieu, sur les parcours de la rencontre, du dialogue et de la liaison avec ses fidèles, ses sociétés et ses élites intellectuelles et religieuses.

Une autre idée majeure que développe cette étude concerne la possibilité de fonder une internationale "pour la dialogue et le rapprochement» entre le christianisme et l'islam, dans un monde saturé de risques d'affrontements et de déchaînements entre civilisations, cultures et religions.

La modification du parcours de l'Eglise catholique vers l'ouverture en direction des autres religions a commencé dès la fin du XIXème siècle, mais n'atteindra son point culminant que dans les années 60 du XXème siècle, et précisément lors de la réunion du second concile pontifical. Outre les multiples évolutions subies par la pensée religieuse occidentale au cours des siècles passés, 
ayant eu une grande répercussion sur la pensée théologique, les hautes autorités de l'Eglise catholique prendront l'initiative de la coexistence créative entre la doctrine chrétienne et les religions mondiales vivantes, en parallèle avec les réalisations du progrès des sciences, du savoir et du système des valeurs.

Le second concile pontifical fut le dernier concile œcuménique réuni par l'Eglise catholique contemporaine, le premier s'étant réuni entre 1869 et 1870 et avait entrepris des pas prudents en direction de la réforme et de l'adaptation aux données de la modernité ascendante. Bien qu'il ne soit pas parvenu à dépasser la reproduction des lois traditionnelles de l'Eglise concernant la foi chrétienne, il posera cependant plusieurs préalables sur lesquels s'appuiera le second concile pour moderniser l'Eglise catholique.

Mais ce qui se déroule dans l'espace catholique au début de la seconde moitié du XXème siècle va effectivement introduire une révolution du savoir dans la théologie chrétienne. Le 25 janvier 1959, le pape Jean XXIII convie les évêques du monde catholique à un concile œcuménique, lui fixant deux buts principaux :

Le premier : renouveler l'église catholique à une époque qui assiste à des changements et des bouleversements rapides, à tous les niveaux. L'Eglise devait trouver un langage adapté à cette époque, un langage associé à des pas pratiques susceptibles de témoigner du christ et du salut qu'il a réalisé...

Le second : consolider l'effort vers l'accomplissement de l'église et propulser le mouvement œcuménique ${ }^{1}$, ce qui signifie, selon le pape, la nécessité de revenir à l'esprit de la foi chrétienne dans le monde, grâce à l'église...

Lors du second concile, qui est celui du renouvellement théologique aux conséquences les plus vastes dans l'histoire de l'Eglise dans son ensemble, la pensée théologique chrétienne et catholique a profondément expérimenté les questions du pluralisme religieux, culturel, social, économique et politique, dans l'univers, et d'après les remarques des théologiens du renouvellement, la question du dialogue entre les enfants de l'univers est issue du cœur même de cette sensibilisation, comme une question essentielle dans le domaine de la foi que le concile s'est appliqué à mettre en œuvre, incitant les catholiques à la pratiquer dans un esprit d'enracinement continu, d'approfondissement permanent,

\footnotetext{
${ }^{1}$ Père Salah Abu Jawde, Jésuite : introduction aux réalités de la foi chrétienne (en arabe), silsilat dirâsât wa wathâ'iq masihiyya, Nº7, Dar Al-Machreq, Beyrouth, 2004, $1^{\text {ère }}$ éd. P. 187.
} 
d'éclaircissement, de fertilisation et d'enrichissement. Et parce que le concile, selon cette évaluation, n'a pas consacré à la question du dialogue religieux ni communiqué, ni déclaration ni paragraphe, cette question s'est trouvée implicite dans la majeure partie des textes. A partir de ces textes, comme le montre leur contexte, il est possible de déduire les principes et les fondements sur lesquels se construit le dialogue religieux dans la pensée de l'église contemporaine, tout comme il est possible de déduire les traits et les particularités de ce dialogue. Il est également possible d'y déceler les buts et les desseins d'un tel dialogue, préciser les problèmes et les questions qu'il soulèvera au niveau de l'expression de l'identité de la foi chrétienne et au niveau de la délimitation de la place théologique qu'occuperont les croyants des autres religions non chrétiennes dans le cercle de la disposition divine plus globale ${ }^{(1)}$.

\section{Modalités du dialogue avec l'islam:}

La théologie catholique contemporaine a accordé au dialogue avec l'islam et les musulmans une place particulière, au point que de nombreux théologiens dans l'église le considèrent comme un devoir religieux devant être appliqué et respecté. Dans cette approche, le deuxième concile du vatican voit dans la «constitution pastorale » «L'Eglise dans le monde aujourd'hui» ce qui suit: «L'Eglise semble être le symbole de cette fraternité qui permet et encourage le dialogue sincère, du fait même de son message qui vise à éclairer tout l'univers par la lumière de l'annonce évangélique, et le rassemblement de toute l'humanité dans un seul esprit, quelles que soient la nation, l'ethnie ou la culture auxquelles elle appartient $»^{(2)}$.

Dans ce texte apparaît clairement le lien étroit entre l'identité de l'église et la nature du message qu'elle délivre en cette période de l'histoire humaine. Selon l'explication théologique, si l'église est le symbole de la fraternité humaine globale, le fait d'entamer avec les autres un dialogue sincère est l'expression la plus noble de l'essence finale de l'église. A partir de cette signification globale et

(1) Mushir Bassil Aoun, fondements théologiques dans la construction du dialogue christano-musulman (en arabe), Dar Al-Machreq, Beyrouth, 2003, p. 69.

(2) Documents du second concile pontifical, l'église dans le monde aujourd'hui (en arabe), parag. 92, trad. Junieh, Liban, 1966. 
ouverte, l'église devient le lieu exemplaire où se manifeste la détermination des gens à se rencontrer, à dialoguer et à faire connaissance ${ }^{(1)}$.

La lecture contemporaine de la conception du dialogue dans l'église catholique atteindra un niveau avancé plusieurs décennies après la théologie des années soixante. Affirmer le devoir du dialogue et de la liaison avec les musulmans mais aussi les fidèles des autres religions est devenu l'une des règles de foi les plus importantes et les plus précises de « la théologie du dialogue et de la connaissance mutuelle ». Pour éviter de considérer que la tenue du second concile pontifical est due aux changements historiques et intellectuels dictés par les conditions d'adaptation au XXème siècle, la théologie contemporaine a jugé que l'insistance sur la nécessité du dialogue ne suffit pas à consolider le dialogue entre le christianisme et l'islam, car pour approfondir le processus de dialogue global, il est nécessaire d'étudier les implications des contenus théologiques des religions chrétienne et musulmane et rechercher les causes théologiques profondes faisant du dialogue islamo-chrétien un devoir religieux inévitable ${ }^{(2)}$. Nous verrons ensuite comment une telle lecture avancera les preuves textuelles et pratiques pour confirmer le devoir de dialogue, que ce soit à partir de l'Evangile ou des résolutions du second concile pontifical. Si nous examinons ce qui est dit à propos $\mathrm{du}$ « devoir de dialogue », nous trouverons le fond de la signification sur laquelle se sont appuyés les théologiens du dialogue pour formuler sa structure cognitive. Dans cette recherche théologique, l'un des points les plus cruciaux est probablement le fait que sa thèse $\mathrm{du}$ dialogue s'appuie sur deux règles fondamentales: la première est celle de la foi évangélique, et la seconde historique, celle de l'église contemporaine catholique, car «l'appartenance à la pensée théologique chrétienne et catholique, constamment à la recherche de renouvellement et de créativité, explique que toutes ces études s'appuient sur une vision théologique que l'église catholique prend pour base pour son enseignement théologique officiel, pour les épreuves existentielles de ses fidèles et pour les tâtonnements de ses grands savants... ${ }^{(3)}$.

Ce point montre la légitimité de la nouvelle parole «du mouvement théologique contemporain », ce qui s'est clairement et indéniablement manifesté

(1) Mushir Bassil Aoun, O.P.cit, p. 14.

(2) Idem, p. 8.

(3) Maurice Bormans, Orientations pour le dialogue entre chrétiens et musulmans (en arabe), trad. Vers l'arabe par Y. Mansour, 21, Manshûrat Al-Maktaba AlBoulisiyya, Beyrouth, 1986, p. 45. 
dans les résolutions du second concile pontifical. Car celui qui s'exprime ainsi le fait de l'intérieur du mental du théologien lui-même et non comme le font des chercheurs qui se situent à l'extérieur des institutions religieuses. C'est ainsi que l'enracinement théorique de la «théologie du dialogue et de la connaissance mutuelle » acquiert, à ce niveau, sa véracité. Ce qui, à notre avis, est important à causes des conséquences du discours sur l'autre, non chrétiens, et plus précisément pour le «tu » musulman, convié à un dialogue en vue de connaître les modalités et les mécanismes par lesquels pensent les pères catholiques, plus particulièrement.

Ce qui rend obligatoire le dialogue dans la pensée théologique contemporaine, institutionnalisé sur l'esprit et le texte du second concile pontifical, est le facteur d'enracinement que vont adopter les responsables intellectuels de l'Eglise catholique. Comme le montre le père Maurice Bormans ${ }^{(1)}$, le dialogue chez les chrétiens est une loi qui plonge ses racines dans le passé, jusqu'à Jésus-Christ lui-même : même s'il a d'abord été envoyé vers « les brebis perdues de la maison d'Israël », il a toujours voulu dépasser les obstacles sociaux, politiques et religieux, il a parlé au Samaritain, a écouté le Syrien Phénicien, a admiré la foi du chef des cent Romains, a loué le pardon « des gens de Ninive » et la sagesse de la reine du sud. C'est pourquoi la parole de Pierre, son premier envoyé, est toujours d'actualité pour les chrétiens contemporains, lorsqu'il dit : « soyez prêts à tout moment à répondre à ceux qui vous demandent la preuve de l'espérance qui est en vous, mais en douceur et respect, et que votre conscience soit droite » (Pierre, 3, 15-16) ${ }^{(2)}$.

D'après les résolutions du second concile pontifical, «l'Eglise incite ses enfants à reconnaitre les valeurs spirituelles, morales, sociales et culturelles des fidèles des autres religions, à les protéger et les développer, et ceci par le dialogue et la coopération avec eux, de manière naturelle et avec amour, associés au témoignage de la foi et à la vie chrétienne dans ce siècle » ${ }^{(3)}\left(\mathrm{N}^{\circ} 2\right)$, mais il faut cependant rappeler, selon les remarques de Bormans, que le dialogue entre chrétiens et musulmans n'a pas franchi les mêmes étapes et ne peut être évalué d'après les mêmes critères en tout lieu et en tout temps. S'ils doivent tous puiser dans les richesses de leurs livres sacrés, dans leur patrimoine et leurs ouvrages ascétiques et soufis, ils savent aussi qu'ils doivent compter sur les facteurs

(1) Idem, p. 46.

(2) Première lettre à Timothée, $4: 2$.

(3) Documents du Second Concile Pontifical, « En cette époque », n², (O.P.cit). 
sociaux, politiques et moraux, idéologiques et culturels qui ont empreint et marqué le tout par la relativité. Tout ce qui sera dit à propos des lieux du dialogue, de ses méthodes, ses écoles théologiques, ses exigences spirituelles, vise en particulier le partenaire chrétien, à qui il est recommandé de ne pas exiger tout cela du partenaire musulman qui demeure libre de voir le dialogue de son propre point de vue. Par contre, ils peuvent tous, par la consultation sincère et la coopération véridique, définir et adapter les exigences du dialogue à leurs situations réelles ${ }^{(1)}$.

\section{Le rattachement du dialogue à la vérité religieuse chrétienne}

Ce qui permet à la théologie contemporaine d'emprunter le chemin du dialogue en tant que rattachement à la réalité religieuse, c'est qu'elle ne prend pas son essort seulement à partir des exigences de la liaison entre groupes humains, mais qu'elle le fait essentiellement à partir de du lien étroit avec les données du nouveau testament. Si la réalité évangélique et la question du salut sont étroitement liées, le dialogue qui suit en est issu : «Dieu veut que tous les gens soient sauvés et atteignent la connaissance du vrai », «celui qui agit dans la vérité accepte la lumière $»^{(2)}$, «Dieu agrée, à tout ce qui se trouve sur terre et dans les cieux, d'être en paix... avec le Christ, qui a confirmé la paix par le sang de sa croix $\gg{ }^{(3)}$.

En méditant sur le secret du salut, apparait, selon la théologie du dialogue lancée par le second concile pontifical, la relation étroite entre le salut et l'avènement du royaume du Dieu, tout comme a fait Jésus-Christ, dans le temps, en Palestine, mais ce royaume qui englobe l'univers, et qui se trouve en même temps à l'intérieur de l'être humain, est le royaume de la paix, de la justice et du pardon, il ne peut y avoir d'équivoque entre lui et toute vérité visible dont il est prisonnier. Et parce qu'il est la vie, comme le montrent les théologiens qui méditent sur le secret du salut, le développement du royaume global de l'univers passe par les étapes du secret pascal, où Jésus a montré sa fertilité globale éternelle, soit la douleur, la mort et la résurrection ${ }^{(4)}$.

(1) Maurice Bormans,O.P. cit, p. 53.

(2) Documents du second concile pontifical...(O.P.cit ).

(3) Ibid ( 20:1).

(4) Bormans, (O.P.cit), p. 53. 
A partir de cette base doctrinaire, les théologiens définissent le dialogue en tant que responsabilité de tous les chrétiens de l'Eglise, par le témoignage, en appelant toute l'humanité à entrer dans le royaume, et à agir en fonction des faveurs que Dieu leur a accordés. Alors, aucune activité humaine ou dialogue religieux, n'est étranger, à leur avis, du royaume de Dieu. Et par conséquent, l'approfondissement de la foi et le renouvellement de l'expression à son propos s'enrichissent par leur rencontre avec les autres cultures, qu'elles soient profanes ou sacrées, anciennes ou modernes, car rien ne peut échapper à la suprématie de l'esprit global de Dieu, où le dialogue qui se déroule dans cette rencontre n'est pas étranger, chez les chrétiens, de la réalisation de l'épanouissement du secret du Christ, du début et de la fin de l'histoire.

Si nous étudions les réalisations des pères contemporains de l'église catholique, nous nous apercevrons comment s'est élaborée la thèse du dialogue à partir de la structure globale des textes du nouveau testament. Nous constaterons ce qu'ils ont montré, que tant qu'il s'agit de la réalité de l'être humain, il n'y a pas moyen d'éviter l'établissement de liens de dialogue. En conséquent, il devient impossible d'imaginer l'existence d'une tradition religieuse - dans la mesure où elle est issue de la réalité existentielle de la présence humaine, et c'est ainsi que Dieu le veut - qui s'éloigne de l'établissement de liens de dialogue. Pour eux, « tant que nous nous attachons à la réalité des traditions religieuses, nous devons dire que le dialogue est devenu indispensable. Et que le principe du dialogue luimême doit être un élément constitutif de la formation des traditions religieuses, de sorte que pour que la religion soit vraiment religion, il faut qu'elle soit religion de dialogue. Si elle ne l'est pas, ou alors elle n'est pas religion, ou alors elle n'est pas conforme aux principes sur lesquels s'appuie la signification de la présence religieuse ${ }^{(1)}$.

Afin d'enraciner la référence de la croyance dans le dialogue, les théologiens du dialogue orientent le contenu de la culture religieuse chrétienne dans ce sens. Par exemple, le pacte avec Abraham (renouvelé au temps de Moïse) entre dans le cadre du pacte global avec Noé. Ce n'est qu'une façon de renouveler le pacte général que Dieu a noué avec toute l'humanité par la création. Dans ce

(1) Wilhelm Dupré, Le dialogue et la vérité, intervention dans la première conférence académique à l'Institut St Gabriel, 6-21 septembre 1992, publiée par Andraos Bachnata et Adel Theodore Khouty sous le titre : la doctrine chrétienne dans sa rencontre avec l'islam, (en arabe), Al-Maktaba Alboulisiya, 2002, p. 82 . 
sens, le pacte avec Abraham se réalise donc pour l'intérêt de toutes les nations. La voix des grands prophètes d'Israël l'a maintes fois rappelé lorsque le pacte risquait de devenir prisonnier d'une nation religieuse extrêmement égoïste. Ceuxlà ont également montré que Jésus-Christ a plusieurs fois affirmé, lors de sa mort sur la croix, qu'aucun être humain n'est écarté du royaume de Dieu : les pécheurs comme les innocents, les samaritains comme les galiléens, le dirigeant des cent romains comme le Pharisee juif atteindront ce royaume unique tant qu'ils demandent le pardon au Seigneur et qu'ils empruntent une nouvelle vie inondée par l'amour de Dieu et des autres ${ }^{(1)}$.

$\mathrm{Au}$ moment où ces théologiens catholiques contemporains tentent de rassembler l'enracinement intellectuel et cognitif et d'inciter les croyants au dialogue et à la connaissance mutuelle, ils entreprennent d'élaborer un texte doctrinaire affirmant que «le chrétien qui doit ressembler, par sa bonté, aux premiers groupes apostoliques, dans sa rencontre avec les autres, quelles que soient leurs différences, sait que le nouveau testament comporte des attitudes pratiques diverses envers les nons chrétiens. Il ne peut qu'y revenir, en fonction des diverses situations qu'il connaît ou des tendances spirituelles qu'ils ressent, passant parfois d'un état à l'autre, ou vivant au même instant, les deux à la fois. Aux côtés de la prédication du salut dans Jésus-Christ, comme l'ont pratiqué Pierre puis Paul dans «Les Actes des Apôtres », qui correspond au devoir de prédication légitime », il y a également le témoignage des chrétiens vivant dans l'exil, qui entendent Pierre répéter : « suivez parmi les nations une voie louable, et lorsqu'ils remarquent leurs actes bénéfiques, ils glorifient Dieu le_Jour du Jugement » (Pierre, 2, 12). Tout comme le christ est "le témoin du Père », les chrétiens sont ainsi invités à être à plusieurs reprises l'évangile et les lettres de Jean. Finalement, il arrive parfois que les chrétiens ressentent ce que Paul ressentait, l'affection qu'il affichait envers ses frères non chrétiens qui insistent à rester tels quels. Aux côtés de «sa peine et sa douleur» à cause de cela, il leur assurait qu'ils bénéficiaient de la sollicitude divine car Dieu ne rejette personne. Ainsi, tant que les voies sont multiples, le chrétien est invité à respecter, à comprendre et apprécier tout choix religieux à cause du secret impalpable que comporte le choix fait par l'homme, car « les actes religieux par lesquels les gens se dirigent vers Dieu, en secret ou en public, s'appuyant sur une décision

(1) Maurice Bormans, (O.P.cit), p. 54. 
personnelle, se situe au-delà, par sa nature, sur tout régime temporel et terrestre » (la dignité humaine) $^{(1)}$.

Ce qui rend ainsi la conception du dialogue, c'est qu'il est institué à partir des expériences de dialogue avec les non chrétiens, après la tenue du second concile pontifical, les théologiens faisant partie de l'Eglise ayant le devoir de formuler leurs recherches et leurs écrits conformément aux nouvelles règles, règlements et méthodes des conciles..

A partir de ces thèses, l'église a placé la détermination confessionnelle, dans le sens de «croyant » en second lieu, après le second concile pontifical, affirmant l'appartenance de l'église au genre humain, avant toute chose, et le lien organique avec l'humanité en général. La déclaration du concile à propos de la relation de l'église avec les religions non chrétiennes « Nostra Aetate » débute ainsi : tous les peuples forment un seul groupe, ont une même origine, le chrétien aujourd'hui réalise qu'il est un membre d'une formidable famille humaine. C'est pourquoi il affirme sa solidarité avec tous les gens, n'apprécie pas le non musulman en tant que «autre » ou «étranger» car il a conscience de cette vérité, que tous les gens appartiennent, par leur libre volonté, même à des degrés divers, au «peuple du Seigneur ». Mais du point de vue de la conscience religieuse, comment peut-on accepter les autres doctrines religieuses, et par conséquent, comment cette conscience peut réconcilier ou concorder ce qui ne peut pas l'être ? En réalité, la pensée catholique essaie de résoudre ce dilemme en produisant deux niveaux ou deux formes de discours, qui sont le niveau humain général et le niveau religieux particulier. Ce discours se base historiquement sur la dualité évangélique connue : ce qui est à César est à César et ce qui est à Dieu est à Dieu » (voir l'Evangile de Marc, 12:17, ainsi que l'évangile de Luc, $20: 25)$. C'est sur cette dualité et définition que s'appuie la nouvelle parole chrétienne (école, scolastique) qui part de principes et thèses «globaux», «totalitaires» et «mondiaux», en vue d'intégrer et de mêler, dans une seule composante, un ensemble de valeurs très différentes. Cette rationalisation menée par les théologiens contemporains de l'idée «d'économie » dans la doctrine, qui remonte à son tour aux thèses de Thomas d'Aquin, où le principe d'indépendance relative entraîne après lui, de

(1) Bormans, (O.P.cit), p. 55 
manière automatique, la reconnaissance juridique du pluralisme des courants et des écoles doctrinaires, et le refus de la thèse qui prétend monopoliser la vérité ${ }^{(1)}$.

\section{La liaison avec les musulmans du monde}

A partir des principes et résolutions fixées par le second concile pontifical, une nouvelle ère théologique catholique a commencé, concernant les rapports avec les religions non chrétiennes, et notamment l'islam. Parmi les principes les plus importants, figure probablement la disposition au dialogue. Le second concile pontifical l'a exprimé dans l'un de ses documents, disant : «conformément à sa mission de soutenir l'unité et l'amitié entre les humains, l'église considère ce qui est commun chez les gens et ce qui est susceptible de mener à leur participation mutuelle les uns avec les autres ${ }^{(2)}$.

Dans leur tentative de former une vision théologique contemporaine pour inclure l'islam dans le cadre du système du salut, plusieurs éminents théologiens catholiques ont présenté ce qui peut être considéré comme une vision religieuse fondatrice du dialogue avec les musulmans.

La plupart de ces tentatives partent de la révision critique et radicale des anciennes conceptions chrétiennes vis-à-vis de l'islam et de ses doctrines, et notamment la vision byzantine rejetant et refusant les doctrines des musulmans. Selon ce qui est communément admis par les récits historiques, ces conceptions s'appuient sur la règle de la polémique et la recherche des outils cognitifs qui prouvent l'invalidité de la doctrine islamique. Ils étaient convaincus que l'islam n'est pas une religion correcte et qu'elle n'a pas une valeur salvatrice. Ils se sont appuyés pour ce faire sur le système de défense que les théologiens chrétiens avaient forgé pour riposter aux objections des adversaires du christianisme, et par conséquent, présenter la preuve de la justesse de la doctrine chrétienne. Ainsi, les théologiens byzantins ont montré, dans leurs ouvrages grecs, que la comparaison entre l'islam et le christianisme éclaire la grande distance qui les sépare, tant dans la doctrine que dans la morale ou les cultes, de sorte qu'il faut considérer que l'islam est une religion erronée et que Mohammad ne peut être, en aucune sorte,

(1) Alexi Jorawski, L'islam et le christianisme, trad. Khalaf Al-Jarad, (en arabe), silsilat 'Alam Al-Ma'rifa, 215, Koweit, Nov. 1996, p. 166.

(2) Adel Théodore Khoury, L'islam dans la vision de la théologie chrétienne, coauteur Andraos Bachtana, dans la collection «christianisme et islam dans le dialogue et la coopération, $\mathrm{n}^{\circ} 16$, Maktaba Boulissiyya, Junieh, Liban, 2002, p. 280 . 
comparé avec Jésus-Christ, c'est pourquoi il faut le considérer comme un faux prophète. Finalement, il s'avère que leur Coran contredit le Livre saint véridique, que Dieu a révélé à Moïse et aux prophètes, aux messagers et auteurs des Evangiles, c'est pourquoi il faut considérer qu'il s'agit d'un faux livre ${ }^{(1)}$.

Cette littérature polémique byzantine à propos de l'islam a laissé un lourd héritage, saturé des causes de rupture et d'invalidation, dues à nombreux facteurs, historiques, sociaux et doctrinaires, mais la formation théologique byzantine se lancera dans ce domaine à partir du point fondamental qui constitue l'élément critère dans la théologie chrétienne dominante au Moyen-âge, le critère du salut. Les Byzantins ont considéré que la religion de l'islam est incapable d'accorder le salut. Pour eux, le salut est tributaire de l'action salvatrice de Jésus-Christ, de sa vie, sa mort sur la croix et sa résurrection. Quant aux moyens du salut, ce sont les secrets de l'Eglise. Et comme l'islam ne croit pas dans l'œuvre salvatrice du Christ, ni dans les secrets de l'Eglise, c'est pourquoi les rites d'adoration islamiques restent incapables d'accorder le salut. Pour eux, ils sont inefficaces, même s'ils ressemblent, en apparence, à quelques rites juifs ou chrétiens ${ }^{(2)}$.

En ces temps modernes, et à cause des expériences profondes vécues par le christianisme avec lui-même, de ses interactions civilisationnelles avec les diverses géographies religieuses, des changements radicaux vont intervenir et culminer dans les années soixante du XXème siècle, avec la réunion du second concile pontifical. Et comme la question du salut des non musulmans avait représenté l'axe des préoccupations de la théologie du Moyen-Âge, la même question a occupé le point central dans la révolution cognitive de la théologie contemporaine.

La vision de la théologie contemporaine sur laquelle s'est basé le second concile pontifical, s'est élaborée comme un champ d'expérience et de réactivité avec les acquis des Lumières, des nouvelles connaissances auxquelles sont parvenues l'histoire comparée des religions et la sociologie religieuse, ainsi que celles des écoles et des courants issus des travaux de l'herméneutique du Livre saint, dans ses deux parties, l'ancien et le nouveau testaments.

L'enracinement théologique du tournant des années soixante s'est opéré sur la base de la description de l'état du monde moderne, où les géographies du monde, civilisationnelles et religieuses, ne sont plus des identités fermées.

(1) Adel Théodore Khoury, idem, p ; 284.

(2) Idem, p. 288. 
Lorsque la théologie chrétienne a pris en compte le pluralisme, elle a dû faire face à un problème extrêmement complexe et important, révélé grâce à la prise de conscience accrue et à la connaissance détaillée des grandes religions, et grâce aussi au contact, large et permanent, entre les fidèles des diverses religions et cultures et des espaces civilisationnels. C'est là que se manifestent, pour les églises, l'ampleur de la vitalité des religions et le rôle décisif qu'elles exercent dans la vie des peuples. La disposition au dialogue est issue de la certitude de l'église de devoir regarder au-delà des divisions qui séparent, vers ce qui est commun entre les gens et les religions, de sorte que les autres religions non chrétiennes sont la référence qui présente aux gens les réponses à leurs questions justes et importantes dans leur vie. Le second concile du Vatican a exposé les questions de la manière suivante : qu'est l'être humain ? Que signifie notre vie et qu'elle est son but? Qu'est-ce que le bien et qu'est-ce que le péché ? Quelle est la source de la douleur et que signifie-t-elle? Par quel moyen parvenir au vrai bonheur? Qu'est-ce la mort, la rétribution et le pardon après la mort? Et finalement, quel est ce secret final qui ne peut être exprimé, le secret de notre vie duquel nous venons et auquel nous nous dirigeons? ${ }^{(1)}$

Dans ses discussions, le théologien catholique Adel Théodore Khoury présente les documents du concile, disant que les religions non chrétiennes ne sont plus jugées indistinctement, comme l'athéisme ou les fausses religions, et par conséquent, leurs enseignements, leurs critères et leurs règlements pratiques ne sont plus refusés dans leur ensemble, car les non chrétiens peuvent parvenir au salut ${ }^{(2)}$, d'après le document du concile, « la lumière des peuples ». Celui qui n'a pas atteint la connaissance vraie, l'Evangile du Christ et son église, sans qu'il en soit coupable, mais recherche Dieu avec un cœur sincère, et tente d'accomplir, par Sa grâce, Sa volonté dictée par sa conscience, celui-ci peut obtenir le salut éternel. La sollicitude divine ne prive pas des choses essentielles du salut ceux qui n'ont pas encore atteint la reconnaissance explicite en Dieu, sans qu'ils en soient coupables mais qui essaient, par Sa grâce, de vivre une vie droite ${ }^{(3)}$.

\section{La centralité de l'islam dans la doctrine du salut:}

(1) Idem, p. 288.

(2) Idem, p. 294.

(3) Idem, p. 295. 
Le discours sur l'islam et les musulmans occupe une place centrale dans les documents du second concile pontifical. Nous pouvons le constater à partir de deux principaux textes :

Le premier : la constitution doctrinaire de l'Eglise, dans lequel nous lisons :

«Il semble que le processus du salut concerne également ceux qui croient dans le Créateur, et en premier lieu les musulmans qui proclament leur foi en Ibrâhim, qui adorent avec nous Dieu l'Unique, le Miséricordieux et le Clément, qui juge les gens le Jour Dernier » (constitution doctrinaire de l'Eglise, parag. 16).

Le second : la relation de l'Eglise avec les religions non chrétiennes, où nous trouvons : «l'Eglise considère avec estime les musulmans qui adorent Dieu l'Unique, le Vivant, l'Immuable, le Miséricordieux, le Puissant qui a créé les cieux et la terre, et tous les gens; ils se soumettent entièrement aux jugements de Dieu, même si Ses Objectifs demeurent invisibles, tout comme Ibrâhim s'est soumis, lui dont la religion musulmane est fière de lui appartenir. Bien qu'ils ne reconnaissent pas Jésus en tant que dieu, ils le louent en tant que prophète, louent sa mère la vierge Marie, l'implorant parfois avec foi. De plus, ils attendent le Jour Dernier au cours duquel Dieu rétribue les gens après leur résurrection. C'est pourquoi ils estiment la vie morale, adorent Dieu par la prière, le don et le jeûne, notamment. Mais parce qu'au cours des siècles, des conflits et des hostilités ont eu lieu entre les chrétiens et les musulmans, le concile invite tous à oublier le passé et à agir avec un effort sincère en vue d'instaurer une compréhension mutuelle, de protéger et de renforcer tous ensemble, en faveur de tous les gens, la justice sociale, les valeurs spirituelles, la paix et la liberté (communiqué sur la relation de l'Eglise avec les religions non chrétiennes, parag. 3).

Dans ces deux textes explicites, la théorisation théologique de l'Eglise catholique va s'approfondissant envers l'islam. Selon cette théorie, l'islam est semblable aux autres religions, il est un préambule au christianisme, du point de vue de l'histoire dans le système du salut divin. Ainsi, l'islam est, du point de vue de l'histoire du salut, parmi les religions qui sont nées avant le christianisme, et dont le parcours salvateur mène jusqu'à la connaissance de la vérité de l'inspiration divine dans Jésus-Christ ${ }^{(1)}$.

Cette interprétation théologique de la place de l'islam dans le système du salut, avec d'autres interprétations parallèles, se retrouvent et se recoupent autour

(1) Mushir Bassil Aoun, (O.P.cit), p. 104. 
d'un point central qui est le paradoxe de la théologie chrétienne contemporaine relative à la pensée exclusive du salut chez l'Eglise, et par conséquent, évitent d'annuler le système doctrinaire de l'islam.

Le théologien Ludwing Hagmann a par exemple indiqué les points qui peuvent constituer des liens entre l'islam et la vérité de Jésus-Christ : « si l'aspect christologique dans la foi musulmane n'a pas atteint sa perfection, mais apparaît imparfaite, nous pouvons considérer cependant l'islam comme un pas dans la direction juste, et une étape sur la voie menée à parfaire la connaissance du christ (voir Le message aux gens d'Afissos 13-4), prenant en considération l'avancée dans trois aspects :

- le passage du fanatisme du sang vers la communauté croyante

- le passage de l'ère de l'ignorance vers celle de la science et de l'inspiration

- le passage du polythéisme vers la reconnaissance ferme en Dieu Unique et Un. ${ }^{(1)}$

Dans le cadre de sa recherche sur "l'islam du point de vue de la théologie chrétienne », Adel Théodore Khoury ajoute les paroles d'un autre théologien, Hans Kung, qui essaie de mettre l'islam en parallèle avec le judaïsme chrétien, le groupe qui ne reconnaissait pas entièrement les enseignements chrétiens sur le christ que le concile macédonien avait finalement proclamé. Du point de vue de la théorie du parachèvement, cela signifie que l'évolution de l'islam en direction du christianisme est possible même si en réalité, l'islam refuse catégoriquement une telle évolution. Dans ce cadre, Khoury reprend ce qu'a répété le théologien catholique Claude Geffré, qu'il est possible de considérer l'islam, dans ses critiques des doctrines chrétiennes, comme une sorte de rappel de la nécessité de protéger la foi contre les anomalies et une incitation à maintenir l'unité. Ainsi, il est possible de considérer le Coran, selon Geffré, comme un des paroles divines adressées aux chrétiens. ${ }^{(2)}$

\section{La rencontre autour de l'Unicité:}

Bien que les textes du concile se soient contentés de définir les lignes générales relatives à l'islam, ils ont cependant ouvert les portes de la recherche approfondie aux théologiens du dialogue dans l'Eglise catholique. Certains contemporains vont même jusqu'à dire que la conception de l'Eglise sur l'Unicité

(1) Adel Théodore Khoury, (O.P.cit), p. 285

(2) (O.P.cit), p. 286. 
de Dieu, l'Unicité de la médiation salvatrice représentée par Jésus-Christ, l'Unicité de l'acte du Saint-Esprit dans l'histoire humaine mais aussi l'Unicité de la médiation de l'Eglise pour montrer la présence de Dieu et la réalisation du Royaume de Dieu, tout cela entraîne à dire que l'Islam ne peut qu'être intégré au christianisme comme la ramification est intégrée à l'origine, la partie au tout, la semence au sol, la branche à la vigne. Par conséquent, l'Eglise catholique dans la théologie des religions qui commence à admettre sa compétence cognitive, reconnaît la religion islamique à la manière de l'intégration, de la complétude et du parachèvement. Elle ne l'éloigne pas du cercle de l'inspiration divine, même si elle continue à considérer que l'inspiration coranique est un champ fertile dans lequel ont été introduites des semences autres que celle du vrai et de la réforme ${ }^{(1)}$.

\section{Le dialogue islamo-chrétien actuel}

La polémique qui a eu lieu au Moyen-Âge entre la théologie chrétienne et la théologie (kalâm) islamique n'a fait qu'approfondir le conflit doctrinaire et donner naissance à la crainte, la méfiance et l'écartement entre musulmans et chrétiens de l'orient arabe. S'il reste encore beaucoup de causes incitant à la polémique, elles sont dues dans leur grande majorité à des influences objectives, historiques et politiques plus qu'au principe de la croyance en Dieu l'Un et l'Unique et en Son Unicité. La controverse de Saint Jean Damascène, par exemple (de la fin du VIIème siècle jusqu'au milieu du VIIIème siècle) à propos des "cent hérésies » dans le « livre des hérésies » a probablement constitué un exemple de rupture dans la polémique doctrinaire entre l'islam et le christianisme à cette époque. Il en est de même pour les controverses du savant Taqî Eddîne Ahmad Ibn Taymiyya le Damascène (1263-1328) en réponse à quelques doctrines chrétiennes, considérant la plupart comme des innovations, disant par exemple que la prière vers l'est n'a pas été ordonnée ni par le christ ni par ses disciples, mais inventée par Constantin. Mais également l'innovation de la croix et des mélodies au cours des prières, ajoutant que la plupart des cultes et des fêtes qu'ils pratiquent n'ont pas été ordonnés par Dieu, etc... ${ }^{(2)}$

Ce qui est commun entre ces deux exemples de polémique dans les théologies chrétienne et musulmane, Jean le Damascène et Ibn Taymiyya, malgré l'écart temporel entre les deux hommes, est qu'ils s'attachent à la lettre et à

(1) Mushir Bassil Aoun (O.P.cit), p. 186.

(2) Aziz Adhame, Recueil de textes du patrimoine (Ibn Taymiyya) (en arabe) Dar Riad Rayyes, Beyrouth, 2000, p. 18-22. 
l'apparence du texte. C'est probablement pourquoi le premier a été décrit par des qualités saintes, comme par exemple le héros de l'église, ou par ce que le pape Jean-Paul II a dit de lui, en réponse au discours du patriarche d'Antioche, Augustin IV Hazim, le qualifiant de « héros et combattant de la foi orthodoxe ». Il en a été de même pour Ibn Taymiyya, que beaucoup considèrent comme le fondateur du salafisme dans l'histoire musulmane. Les paroles d'Ibn Taymiyya dans le contexte de la lettre à Serge, roi de Chypre : « je suis un député du christ et des autres prophètes quand je conseille le roi et ses compagnons et leur recommande le bien » montrent probablement le degré de sa fierté et notamment lorsqu'il dit, dans son message au sultan Al-Nasir Mohammad b. Qalawoun qu'il est le rénovateur de la communauté, à la fin du VIIème siècle ${ }^{(1)}$.

Les retombées de la polémique théologique traduisent clairement les conséquences de la coupure entre musulmans et chrétiens. A cause de cette polémique, les possibilités de dialogue semblaient maigres, sinon impossibles tant que les conflits défensifs prenaient le dessus, avec des fréquentes attaques contre l'autre, relatives à sa croyance et sa foi. Mais un nouveau parcours dans la vision réciproque entre les institutions religieuses, chrétiennes et musulmanes, a pris le relais pour affirmer la nécessité de remplacer la relation de polémique et de coupure par une relation de dialogue et de liaison. Les deux parties ont considéré qu'il n'était pas permis de poursuivre la situation telle quelle, à partir de la thèse suivante : lorsque l'autre te cherche conflit dans ta religion et ton identité utilisant sa religion et son identité comme arme, sur laquelle il s'appuie en t'attaquant, il est naturel que la zone la plus sensible et la plus aigue en toi, dans ta religion et ton identité soit mobilisée et que le fanatisme, la violence et l'écartement se forment dans un renouvellement incessant. Si l'identité et la religion font partie du cadre dénommé par le stratège français Jean Guitton la métaphysique politique, il est inévitable que les musulmans et les chrétiens politisent la religion pour servir les intérêts politiques et économiques de telle ou telle partie.

Le concile réuni entre le début et le milieu des années soixante constitue un tournant historique dans la relation entre l'Eglise catholique et les musulmans et dans leur compréhension de l'islam. Le document émanant du second concile pontifical à ce moment instituera probablement une nouvelle relation avec les sociétés islamiques notamment, et avec les autres religions non chrétiennes de manière générale.

(1) Idem, p. 24. 
Il y a donc, entre chrétiens et musulmans, de nombreux points communs sur les plans de la foi et de la morale. Sur le plan de la foi, les chrétiens et les musulmans s'accordent sur l'adoration de Dieu le Créateur, l'Unique, qui s'est adressé aux gens par le biais des prophètes, depuis Ibrâhîm jusqu'à Jésus, ils rendent hommage à Marie la vierge, ils implorent la résurrection des morts. Sur le plan de la vie religieuse et de la morale, ils s'accordent sur la prière, le don, le jeûne. Nous pouvons ajouter que l'accord comprend également de nombreux comportements moraux ${ }^{(1)}$.

Si pour l'Eglise catholique contemporaine, le dialogue consistant à se connaître mutuellement fait partie des fondements de la foi et du système doctrinaire, il représente, dans l'espace de l'islam, un des piliers de la relation avec autrui. Comment apparaît cette relation à autrui dans les motivations de la loi islamique?

\section{Le devoir de faire connaissance dans I'Islam:}

La théologie chrétienne novatrice n'a laissé aucune occasion en parlant du lien entre l'inspiration et le temps humain sans se tourner vers le Livre saint qui incite au dialogue, comme nous l'avons vu. Quant à l'espace intellectuel musulman, le dialogue pour le rapprochement, comme nous le verrons, a le statut de devoir. Sans ce dernier, le message divin n'est pas transmis, comme l'indiquent le Coran et la sunna.

Nous constaterons dans ce domaine comment le dialogue fait clairement partie du texte coranique. De nombreux lecteurs de l'inspiration sont même allés jusqu'à expliquer et interpréter le saint Coran pour dire qu'il est le livre du dialogue par excellence. La conception coranique du dialogue découle du décret divin sur la diversité de la création, cette diversité, cette variété et ce pluralisme sont imposés par la multiplicité de la création qui est la loi du jugement de Dieu dans la création.

Le saint Coran a décrété que la différence des gens, dans leurs doctrines, communautés et opinions, est une loi immuable qui ne changera pas. Cette différence ne sera abolie qu'après la disparition de la vie sur terre. C'est ce qu'indique la Parole du Très-Haut: "Or ils ne cessent d'être différents à l'exception de ceux auxquels ton Seigneur a accordé Sa miséricorde. Et c'est

(1) Voir le document du Second Concile Pontifical, Constitution doctrinaire de l'Eglise. 
bien pour être si différents qu'Il les a créés " (Hûd, 118-119). "Certes, ceux qui croient, ceux qui pratiquent le judaïsme ainsi que les Sabéens, les chrétiens, les zoroastriens et les polythéistes, Dieu les départagera le Jour de la Résurrection, car Il est témoin de toute chose " (Al-Hajj, 17).

Sur cette base coranique de la différence, l'Islam a légiféré le droit des religions de la révélation qui lui sont antérieures. Lorsque la loi Mohammadienne a pénétré dans le cœur du temps et de l'espace, elle n'a pas nié les religions, ni s'est comporté avec elles par la force, la contrainte ou l'obligation, sur la base même de la distinction entre la vérité et l'erreur (Al-Baqara, 256) et la nécessité de choisir la croyance ou de suivre la communauté. C'est ce qu'affirme le Livre divin dans le verset «pas de contrainte en religion, la vérité s'est distinguée de l'erreur ». C'est également ce que le prophète, paix et saluts de Dieu sur lui, dit, inspiré par Dieu le Très-Haut : " Rappelle-leur tout cela ! Car ton rôle se limite à le leur rappeler et tu n'as sur eux aucune autorité " (Al-Ghâshiya, 21-22), ce qui ne nie pas la nécessité d'appeler à la croyance dans le Vrai, en Dieu, et à suivre la religion finale de l'islam.

Bien que l'islam ait apporté la dernière doctrine des messages célestes, l'orientation coranique a veillé à inciter les musulmans à ce que l'appel à Dieu soit associé au dialogue avec ceux qui professent une doctrine différente: "Appelle à la voie de ton Seigneur avec sagesse et par de persuasives exhortations » (Al-Nahl, 125). Le Coran a accordé une place particulière aux gens du Livre en disant : "Ne discutez avec les gens des Ecritures que de la manière la plus courtoise " (Al-'Ankabût, 46), ce qui signifie en réalité ôter à la discussion son contenu contraignant, pour qu'il soit un dialogue se déroulant dans une foi commune en l'Unicité de Dieu le Très-Haut. Les sources et les références religieuses islamiques ont présenté une image positive du christianisme et du judaïsme, les décrivant comme des religions célestes qui partagent avec l'islam la foi dans les prophètes, les messagers, le Jour Dernier et qui s'élancent du cercle de 1'Unicité(1).

Les responsables et les savants musulmans considèrent la multiplicité dans l'inspiration divine comme une loi de la création, comme nous l'avons indiqué. Ils s'appuient sur la vérité affirmant que la guidance divine est la première source de l'organisation humaine. La guidance divine, telle qu'elle est montrée dans les

(1) Anouar Abu Taha, le dialogue islamo-chrétien, dans «le discours islamique, jusqu'où ? », Dar Al-Fikr, Damas, 2006, p. 63. 
explications islamiques, se déroule selon deux voies: relative à l'acte de la création et législative. La guidance relative à l'acte de la création est celle qui englobe toute l'existence puis s'étend pour inclure tout ce qui existe et tous les êtres vivants, et par conséquent, toute l'humanité. Cette guidance est liée aux questions non expérimentales, dans le sens où elle ne fait pas partie du cadre de «la volonté des existants», comme par exemple: l'évolution naturelle des existants fait partie de la chaîne de la guidance relative à l'acte de création.

Quant à la guidance législative, elle est un don de Dieu à l'humanité par le biais des prophètes et des messagers. Elle se manifeste dans la réalité, dans le sens où elle éclaire le chemin vers l'Unicité, ce qui signifie qu'elle fait parvenir à la destination voulue ; il faut, pour ce faire, agir selon les ordres divins, par choix et en toute volonté. Selon une explication concise de certains ulémas musulmans : Dieu le Très-Haut accorde aux croyants qui s'engagent dans la guidance législative une sorte de guidance relative à l'acte de création qui signifie faire parvenir à la destination voulue (et notamment au cours des étapes de la marche et du parcours vers le Vrai Ultime). La guidance législative signifie donc que Dieu le Très-Haut procure aux gens la loi qui leur assure le bonheur et les rend conscients, grâce à l'ordre de faire les actes vertueux et de s'interdire les mauvais, afin qu'ils choisissent la route de leur choix, pour parvenir à la destination qu'ils souhaitent, en toute liberté.

Les religions monothéistes comprennent ces deux sortes de guidance divine, même s'il y a une différence dans l'interprétation et l'explication, chez les savants musulmans, les théologiens chrétiens et les juifs. Mais ce qui est commun et ce qui est différent demeurent le principe régissant la conception coranique, car ce qui est commun au niveau de l'acte de création est stable sur la base de l'Unicité, alors que ce qui est différent est une chose possible dans les domaines de la légifération. C'est pourquoi le saint Coran insiste sur la maîtrise du dialogue, de la discussion et de la polémique dans un espace de miséricorde mutuelle. La polémique dans le sens de l'échange de la parole entre l'Islam et les gens du Livre doit s'appuyer sur la connaissance et se déroule sur la base de la science. On ne peut se diriger vers la polémique ou vers la discussion qu'après s'être assuré de la preuve, avoir acquis la science et découvert le vrai. Nous nous arrêterons à certains versets coraniques qui ont trait à la bienséance de la polémique ${ }^{(1)}$ :

(1) Mahmoud Haidar, la nécessité de connaître le Coran, conférence à l'université st Joseph, Beyrouth, 15/3/2008. 
"N'insultez pas ceux qui invoquent d'autres divinités que Dieu, car ils seraient tentés, dans leur ignorance, d'insulter à leur tour Dieu. C'est ainsi que Nous embellissons aux yeux de chaque peuple ses propres actions. Puis ils retourneront tous à leur Seigneur, qui les informera de leurs actes passés " (AlAn'âm, 108).

"Tel parmi les hommes discute au sujet de Dieu sans détenir la moindre connaissance, et se laisse entraîner par tout démon rebelle " (Al-Hajj, 3).

"Certes vous pourrez discuter des choses que vous connaissez. Mais pourquoi disputez-vous de celles dont vous n'avez aucune connaissance? Dieu sait; mais vous, vous ne savez, pas " (Âl-'Imrân, 66).

A partir de cette vision, empreinte de miséricorde, du dialogue et de la discussion, le noble Coran a présenté le christianisme comme une pure religion céleste, rapportant la vie et le message du christ et l'honorant, ainsi que sa mère Marie, de la manière la plus noble. Dans le texte coranique, Marie, la pure vierge, a été choisie par Dieu le Très-Haut parmi les femmes des mondes. Elle a été le lieu de l'annonce de l'arrivée du christ dont la naissance a été un miracle divin. Dans le Coran, également, 'Issa, le christ, est un prophète, un messager, la parole de Dieu et une âme provenant de Lui. Il est l'auteur de miracles importants que Dieu lui a accordés : il parle à Dieu dans le berceau et ressuscite les morts par Sa volonté. Il est décrit par la guidance, la lumière, celui qui a attesté la Bible, le bon conseil aux croyants et le Coran l'a appelé le livre. Ses disciples ont été décrits par la foi, la compassion, la miséricorde, la clémence et l'obéissance au prophète de Dieu. De même, le Coran a loué les croyants parmi les gens du Livre en plus d'un lieu, indiquant leur rang, leur justice, leur foi et leur crainte de Dieu le Très-Haut, ainsi que leur modestie vis-à-vis de Lui.

Ainsi, l'image du christianisme exposée par l'inspiration divine dans le Coran est celle d'un christianisme qui n'est pas objet de dialogue ou de discussion négatives, car la foi en lui fait partie de la foi musulmane, qui l'atteste et l'englobe sur la base de la miséricorde divine, Dieu ayant ordonné d'y croire comme partie de la croyance dans les messagers de Dieu. Il en est de même pour les prophètes des fils d'Israël.

Quant au dialogue et aux discussions, le noble Coran a distingué entre la première image originelle et l'image historique, du christianisme. La discussion a été menée à propos d'un ensemble de questions doctrinaires, comme celle de la 
déification, de la crucification, de l'esprit saint et celle de prendre les prêtres et les moines pour maîtres en dehors de Dieu, etc...

Mais aux côtés de la discussion, le noble Coran a fixé les règles du dialogue miséricordieux dans le cadre de l'espace historique, que nous pouvons rassembler sous deux règles principales :

La première : la foi en Dieu l'Un, l'Unique

La seconde : l'appel au dialogue en vue de construire une société croyante et morale, dépourvue d'injustice et de corruption. C'est ce qu'indique le verset suivant : «Ô gens des Ecritures ! Mettons-nous d'accord sur une formule valable pour nous et pour vous, à savoir de n'adorer que Dieu Seul, de ne rien Lui associer et de ne pas nous prendre les uns les autres pour des maîtres en dehors de Dieu ». S'ils s'y refusent, dites-leur : Soyez témoins que, en ce qui nous concerne, notre soumission à Dieu est totale et entière » (Âl-'Imrân, 64).

Le messager de Dieu a recommandé et adopté des positions inspirées de la révélation divine et ses applications historiques. Nous rappelons sa rencontre avec la délégation de Najrân qui a discuté avec lui à propos de plusieurs questions sans cependant croire. Malgré cela, les versets ont été révélés à propos de cette discussion. Lorsque la rencontre tirait vers sa fin, le messager de Dieu leur a rédigé une promesse et une assurance sur eux-mêmes, leurs terres, leurs biens et leur religion, devant témoins. Il y a également ses messages à plus d'un roi chrétien, où un pacte a été signé par les deux parties, où on y lit : «A Najrân et leurs suites, la proximité de Dieu, la parole de Mohammad, le prophète et messager de Dieu, sur eux-mêmes, leur communauté, leurs terres et leurs biens, leurs absents, leurs présents et leur allégeance et leurs prières, nous ne changerons aucun prêtre ni moine de leurs prêtres ou de leurs moines. » Nous devons ajouter, parmi les paroles du messager de Dieu, la recommandation relative aux gens protégés, disant: «je vous recommande sous la protection de Dieu, c'est une protection de votre prophète », et : « celui qui tue une âme ayant pactisé ne sentira pas l'odeur du paradis » et «si vous conquérez l'Egypte, comportez-vous avec bonté avec les Coptes, ils sont sous protection et miséricorde... ».

Il nous reste à dire que la vision coranique de Dieu et du monde est de montrer, par la parole divine, la science de Dieu de la création. C'est pourquoi la compréhension du texte sacré, que ce soit dans la lecture explicative ou dans la lecture interprétative, est fondée sur les règles de la raison, de la logique, de la preuve et de la foi. Parce que ces fondements sont en faveur de l'être humain que 
Dieu le Très-Haut a honoré en en faisant Son successeur, le dialogue créatif entre les gens prend un aspect de devoir, inclus dans les buts et les objectifs du texte sacré.

L'expérience historique de l'islam et de la société musulmane au temps du prophète et après lui, a été une fidèle et sérieuse application de la légifération dans le domaine du dialogue et de la coexistence, illustrée par l'expérience du prophète avec les juifs et les chrétiens dans Médine, avec Najrân et les autres, en passant par toutes les étapes historiques où la société musulmane vivait une cohésion sur les bases de l'islam.

L'indulgence issue de la centralité du dialogue dans l'islam ne se limite pas aux questions de la foi et de la doctrine, mais elle pénètre l'espace civilisationnel et humain dans ses multiples aspects. Abordant la polémique de l'islam, certains penseurs musulmans contemporains insistent sur le principe du dialogue avec l'autre, dans cette polémique. Le dialogue est un concept élaboré par le Coran d'abord dans la civilisation islamique, l'entérinant dans la vision globale des musulmans, pour en faire une partie de leur construction mentale et psychologique, de sorte qu'il n'est plus possible d'envisager de se passer de lui, quels que soient les aspects de la pensée, de la conception ou du comportement ${ }^{(1)}$. C'est sur ces vérités centrales qu'insistent ceux qui parlent de dialogue. Le dialogue dont il s'agit aujourd'hui, qu'il soit au niveau des religions ou des civilisations, est un dialogue équilibré et tolérant au premier niveau, qui vise à la connaissance par les croyants des bases sur lesquelles s'instaurent les doctrines de l'unicité dans le christianisme et dans l'islam, en vue de consolider les valeurs de la foi et de la morale, et au second niveau, dans le but de dialoguer pour bâtir des ponts équilibrés et équivalents entre les civilisations islamique et occidentale, en rejetant l'idée de domination et de dépendance, mais aussi la peur, la méfiance et le complexe de la collision. Cette possibilité de dialogue est effectivement présente, notamment parmi les penseurs en Occident. Ce qu'a exprimé Teitler, disant que le dialogue est un moyen de «convaincre », teinté de dignité, dans le rapport avec toutes les parties, même si elles sont en désaccord. L'intérêt commun général gît dans la recherche de la vérité, autant que possible et autant que la raison puisse y parvenir, dans un climat de confiance et de respect mutuel ${ }^{(2)}$.

(1) Imam Mohammad Mahdî Shamseddîne, « sociologie politique islamique » (en arabe) $\mathrm{N}^{\circ} 1414$ h. 1994, p. 101.

(2) Taha Jâbir Al-Alwânî, particularités et mondialité de la pensée islamique contemporaine (en arabe), Dar Al-Hadî, Beyrouth, 2003, p. 125. 
Ce qui a rendu créatif le dialogue de la connaissance mutuelle entre christianisme et islam est probablement le sentiment qu'il s'agit d'un devoir, confirmé par les objectifs de la foi et de la morale, dans les deux religions.

Si des obstacles se sont dressés, historiquement, face à ces dialogues, ils sont dûs aux longs affrontements entre les sociétés islamiques orientales d'une part et les ambitions des Etats impérialistes occidentaux, depuis les croisades jusqu'à présent.

Sur ce problème précis, il semble que la recherche sur une zone de connaissance mutuelle pour comprendre profondément ces considérations demeure exceptionnelle, alors que cette zone est supposée, en premier lieu, séparer entre l'espace religieux et celui des intérêts géo-stratégiques et empêcher leur collision de maitriser les relations entre l'orient islamique et l'occident chrétien.

Reste la question de savoir s'il y aura, à l'horizon, ce qu'on peut appeler "l'internationale du dialogue de connaissance mutuelle» entre les hautes instances religieuses qui proclament l'Unicité mais aussi entre les forces de la société civile et les élites religieuses, intellectuelles et culturelles en orient et en occident, de façon à reconsidérer le système des valeurs et de la morale dans le monde, pouvant contribuer de manière effective à instaurer un équilibre créatif, avec les centres de décision internationale, pour empêcher les guerres, combattre les épidémies et protéger le droit de l'être humain à la vie et l'existence.

C'est en fait la question de référence à laquelle il faut répondre au début du XXIème siècle. 
